\title{
Towards deeper comprehension in higher engineering education: rethinking "in theory yes, but not in practice"
}

\author{
Aki Korpela ${ }^{1}$, Timo Tarhasaari², Lauri Kettunen², Risto Mikkonen² and Hanna Kinnari- \\ Korpela ${ }^{1}$ \\ ${ }^{1}$ Department of Electrical Engineering, Tampere University of Applied Sciences, Tampere, Finland \\ 2 Electromagnetics, Tampere University of Technology, Tampere, Finland \\ For correspondence: aki.korpela@tamk.fi
}

\begin{abstract}
In the economic pressure of recent decades, many universities have invested in increasing the number of accessed and graduated students. However, this has led to another problem: there is not enough higher learning in higher education. According to generally accepted view, the problem lies in the eagerness of overplaying immediate skills in engineering education. To manage with the situation we propose that special attention is paid to logical reasoning behind the models employed in elementary engineering education. The idea is based on the hierarchical nature of physics, which enables a consistent method to avoid the risk of overplaying the immediate skills. In this paper, we seek for higher learning by proposing such a method and presenting a case study of solar electricity. When modelling the nature, on each level of hierarchy certain laws of physics lay the foundation for explanations there. We call them the cornerstones of modelling. Due to the hierarchical nature of physics, the cornerstones on a certain level of hierarchy are only built from the ones on the lower level of higher abstraction. When we stand above the cornerstones, they appear as unchallenged rules. But most importantly, if we take a hierarchical step towards fundamental models and look underneath the cornerstones, instead of unchallenged rules, they appear as testable properties. This epiphany about the hierarchy of physics enables a consistent method for deeper understanding in education. In the end, we seek for higher learning by challenging the meaningfulness of a common utterance "in theory yes, but not practice".
\end{abstract}

Keywords: higher engineering education, higher learning, natural sciences, cornerstones of modelling, electrical engineering

\section{Introduction}

University education has recently confronted an emerging problem with learning. Keeling and Hersh (2011) condense this in a rather strong statement: "There is not enough higher learning in higher education." Also others, such as the Carnagie Foundation, have raised a similar kind of concern (Sullivan 2008; Sheppard 2008). While the worry on higher learning has become topical, the global economic pressure has also raised the awareness of costs and effectiveness. This results in a dilemma; universities are concerned of the learning of their existing students and simultaneously they feel a pressure to become more cost effective.

In the end of the day, investments in higher education make sense only if students learn. Higher learning is a necessity for scientific research and competitive R\&D. Consequently, if there are significant deficiencies in learning, a long term economical threat to the society is created. Accordingly, in the long run nobody gains by false shortcuts in higher education. (The Economist 2012; Bok 2003; Rosovsky 1990)

Although the applications of science constantly expand and diversify, the logical abstract structures of fundamental scientific models remain the same. Since the very purpose of science is to understand 
and explain the world with as small amount of principles as ever possible - instead of listing all possible findings and observations - the roots of higher learning are closely related to understanding the basics exploited to explain the countless number of details.

This is also the reasoning of Anderson (1972) who divides the development of science into intensive and extensive research. Intensive research goes for the fundamentals, whereas the extensive work exploits the known fundamental models in the explanation of the world around us. Although such a distinction may sometimes appear ambiguous, the majority of research is always extensive. For, the discovery of new fundamental models tend to increase the activities in understanding previously unexplained phenomena. Education also typically utilizes the extensive research, and one of the key issues behind this paper is: also the intensive approach needs to be introduced to the students.

In natural sciences, the practice of analyzing a complex phenomenon in terms of more fundamental ones is known as reductionism (Polkinghorne 2002). The idea of reductionism in physics is based on the hierarchical nature of natural sciences. That is, the cornerstones of modelling on a certain level of hierarchy are only built from the ones on the lower level of higher abstraction. For example, in basic circuit analysis of electrical engineering the cornerstones of modelling are Kirchhoff's laws and the generalized Ohm's law. When a hierarchical step towards fundamental models is taken, these three cornerstones are only built from Maxwell's equations and constitutive laws. Through the history of modern education in physics, the power of reductionism has been widely accepted in the teaching of basics (Feynman 1963; Tinkham 1975). Reductionism does not bias mathematical formulas, but instead favors plain words. For a teacher reductionism is a tool to emphasize the main concepts, but through the requirement of strong content knowledge, it is also a rather demanding method. There is a reason why L.S. Shulman (1972) has strongly advocated: "Those who can, do. Those who understand, teach."

\section{Dilemma between immediate and long-term needs}

For an individual higher education is a long-term investment. The very idea is, the time invested in learning will later on pay back as one gains skills and expertise for tasks which are not possible to master without the education. Obviously, it is also in the interest of the society to support this kind of activity.

The remaining question is, how quickly the investment should pay back to an individual and to the society. This is a very actual issue especially in engineering whose emphasis is on getting devices, machines and systems to run. Understanding why something works is typically only a secondary priority. For this reason the training of engineers lends itself to involve into teaching lists of formulas, rules, standards, and descriptions of measurement procedures. There are satisfactory tools in achieving quickly the desired result although they are inaccurate from the scientific point of view. Consequently, the more the education biases immediate needs, the bigger the risk to result in a workmanship that resembles a cookbook of weakly interconnected recipes, and where the level of professionalism becomes measured by the length of list of recipes. It is clear that competitive R\&D cannot be based on such a cookbook. This kind of approach does not fully utilize the power of science making it possible to understand more from less. Hence, the immediate needs should not be the driving force behind higher education.

The practical challenge in all teaching and learning is to find a reasonable balance between the immediate and long term goals. The optimum is not at all a trivial issue. In a famous experiment of teaching elementary school mathematics from 1930's, L. P. Benezet abandoned all formal instructions in arithmetics below the seventh grade. Instead, he concentrated on teaching the children to read, to reason and to recite. This is to say, he neglected the idea that children's progress should be measured 
systematically after some weeks. Surprisingly enough, the conclusion after the experiment was that the group of children eventually outdistanced the pupils under conventional education also in mathematics. (Benezet 1935; Benezet 1936)

The celebrated mathematician, H. Whitney, who got inspired by Benezet's work, concluded: "Children under school-aged learn in manifold ways with no formal teaching, at a rate that will never be equaled in later life. Yet the same children find much simpler things far more difficult as soon as they are formally taught in school" (The New York Times 1986). Whitney's idea was that children should be challenged to discover things rather than simply taught them to accept rules. He passionately pronounced that the poor results of mathematics education are mainly due to the methods of teaching, not due to the incapability of children (Whitney 1985; Whitney 1987). In other words, in the worst case formal teaching methods block children from learning.

In higher education of engineers we deal with the same issues. The problems of learning and teaching in elementary school mathematics remain in higher education as well; formal methods based on accepting rules produce unsatisfactory learning. Instead, learning should involve a strong component of absorbing properly the basic reasoning (Wieman 2013). This all is closely related to the hierarchical nature of physics. When students are given a clear view about the reasoning of the modelling cornerstones, at the same time they are offered a great opportunity for deeper understanding. And in the best case, the fear related to the learning of physics mitigates, when fuzzy rules are simply seen as testable properties. The role of a teacher is essential here: if a teacher has any fear towards the learning of physics or mathematics, it may unintentionally be inherited to students also.

In this paper we seek for methods to gain more profound understanding, which also is a sufficient condition to achieve the highest educational objectives defined in Bloom's taxonomy (Bloom 1956). In order to teach a topic on a certain level of hierarchy, one should be able at least to test the validity of cornerstones on that level. Thus, a hierarchical step towards fundamental models has to be taken in order to see underneath the cornerstones.

All this adds up in saying that the fundamentals need to be introduced to students with a great care. Generally, regardless of the educational level, it is on the teacher's responsibility to condense essential substance for students. In this paper we say that while teaching the fundamentals, it is highly important to concentrate on the careful reasoning behing the cornerstones of modelling. Higher education needs to find ways to efficiently convey the basic fundamentals to students; simplification of the abstract material and exemplification require great care. The topical issue is to make sure the economic pressure does not get out of control pushing universities to bias immediate needs and goals in cost of higher learning.

\section{Case study: operation of solar cell}

In order to clarify the main idea of the paper, we'll study a concrete topic in two different ways. As solar energy is globally a topical issue, we'll investigate the operation of conventional silicon solar cell. The objective is to determine the solar cell load characteristic. Here, the technical issues will be kept as simple as possible in order to emphasize the differences in educational methods. First, we'll study the problem with a circuit analysis model by using the cornerstones as unchallenged rules. Then, a hierarchical step towards fundamental models is taken in order to see underneath the cornerstones. Thus, they are considered as testable properties. Although the latter educational method is slightly more time consuming, both of them are still applicable to the same basic course on solar cells. 


\section{Approach of unchallenged rules}

In order to concentrate on the educational aspects, we'll keep technical details as simple as possible. Consequently, the solar cell is modelled here with a simple circuit presented in figure 1 . Thus, the model includes an ideal current source connected in parallel with a diode. Keeping in mind that the goal is to determine the load characteristic, we have to study the interdependence of voltage $V$ and current $I$, when an adjustable resistor $R_{\mathrm{L}}$ is connected between the nodes in figure 1 . Ideal current source, $I_{\mathrm{sc}}$, represents a constant short circuit current of a solar cell. And if we use the ideal Shockley equation for the diode, its current can be written as

$$
I_{\mathrm{pn}}=I_{\mathrm{s}}\left(e^{V / V_{\mathrm{T}}}-1\right)
$$

where $I_{\mathrm{s}}$ is the dark saturation current of a pn junction, and $V_{\mathrm{T}}$ is so called thermal voltage. The current of solar cell, I, can now be derived from Kirchhoff's current law as

$$
I=I_{\mathrm{sc}}-I_{\mathrm{pn}}
$$

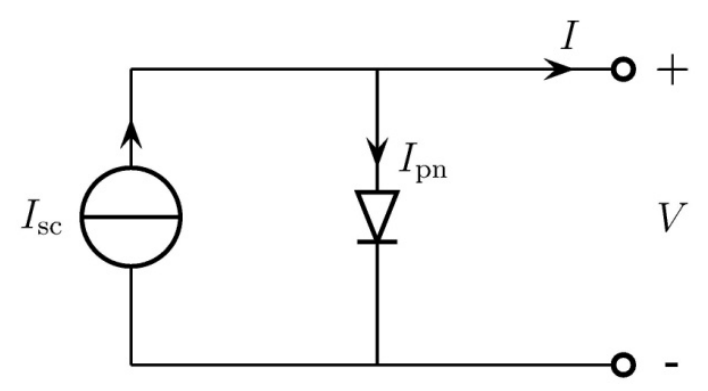

Figure 1. Simple circuit model for solar cell.

And by combining equations (1) and (2), we get

$$
I=I_{\mathrm{sc}}-I_{\mathrm{s}}\left(e^{\mathrm{v} / \mathrm{V}_{\mathrm{T}}}-1\right)
$$

In fact, Shockley diode equation (1) and Kirchhoff's current law (2), are the cornerstones of modelling in this circuit analysis approach. Here, they are taken as unchallenged rules that lay the foundation for explanations.

In order to increase clarity, let's give some typical values for the variables. In a midday sunshine with the solar cell temperature of $298 \mathrm{~K}$, the following values are valid: $I_{\mathrm{s}}=10^{-10} \mathrm{~A}, I_{\mathrm{sc}}=8 \mathrm{~A}$ and $V_{\mathrm{T}}=25.85$ $\mathrm{mV}$.

Now we are ready to study the $I(V)$-characteristic of a solar cell. As a starting point, let's consider two special situations which produce the maximum values for current and voltage: short circuit and open circuit, respectively. In the first case, the nodes in figure 1 are in short circuit resulting in $R_{\mathrm{L}}=0 \Omega$ and $V=0 \mathrm{~V}$. Now, due to the path of zero resistance between the nodes, the maximum current $I_{\mathrm{sc}}=8 \mathrm{~A}$ flows across the load. In the open circuit situation the load resistance approaches infinity resulting in $I$ $=0 \mathrm{~A}$. Now the voltage $V$ has its maximum value $V_{\mathrm{oc}}$, which can be derived from (3):

$$
V_{\mathrm{oc}}=V_{\mathrm{T}} \ln \left(\frac{I_{\mathrm{sc}}}{I_{\mathrm{s}}}+1\right)
$$


With given values substituted in (4), the open circuit voltage is $0.649 \mathrm{~V}$. Now we have the end points for our $I(V)$-curve in figure 2. But how do we explain the behaviour between them?

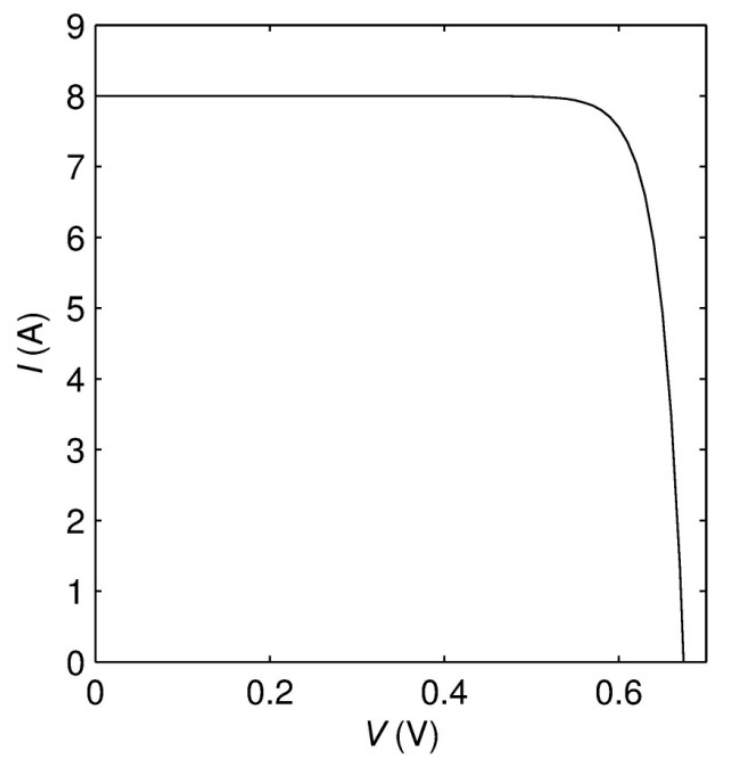

Figure 2. Current-voltage-characteristic of solar cell.

The $I(V)$-behaviour between the end points can be explained with equation (3). Thus, it is the property of the exponential function in Shockley equation. By using a circuit model approach and its cornerstones, we have now been able to explain the load characteristic of a silicon solar cell. By making the circuit model more complicated, the real $I(V)$-curve can be modelled more precisely. However, the superficiality still remains. In fact, with the circuit model we are not truly modelling the physical phenomena taking place in a solar cell. Instead, we are only imitating its black-box behaviour. In order to gain a deeper understanding of related phenomena, we have to look underneath the cornerstones. However, in this approach of unchallenged rules, we stood all the time above the cornerstones. No gestures were made to test their validity.

Approach of testable properties

Now we'll slightly expand our view on solar cell load characteristic. The main substance will remain exactly the same as in the approach of unchallenged rules, so both the educational methods here are suitable for the same basic course on solar electricity. However, an additional lesson is needed to introduce the students the idea of cornerstones as testable properties. The aim is to look underneath the cornerstones in order to see their prerequisites. Thus, we also need to take a hierarchical step towards fundamental models, since the new cornerstones of the lower level have to be identified. As the hierarchical step is taken, at the same time the level of abstraction increases. However, we are not trying to formalize the cornerstones. Instead, we only seek for the sufficient conditions that validate their utilization. When we take a hierarchical step towards fundamental models from the circuit model approach, we split Kirchhoff's current law and Shockley diode equation into more delicate models. The results are presented in figure 3, where the level of higher abstraction is called as solid state physics approach. 


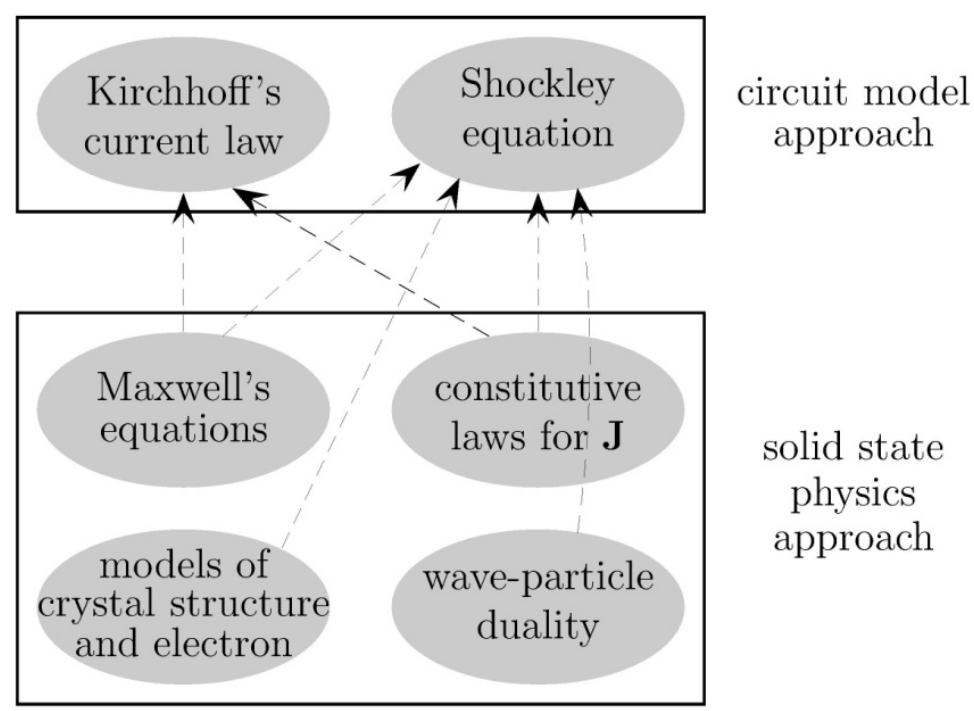

Figure 3. Cornerstones of modelling in circuit model approach and in solid state physics approach.

Thus, as we only want to expand the view given by the approach of unchallenged rules, all the explanations and equations already presented are also valid here. But, in addition, we seek for deeper understanding by considering the cornerstones as testable properties. They are not unchallenged rules anymore, but instead, we try to find the conditions for their validity.

When we look underneath the cornerstone of Kirchhoff's current law, we are dealing with Maxwell's equations. In fact, Kirchhoff's current law originates from the principle of charge conservation with the assumption of sourceless current density. In addition, also the constitutive laws for current density have to be included, since we assume that current flows only in conductors. The splitting of Shockley equation is a bit more complicated. In order to understand its foundation, we need Maxwell's equations, the constitutive laws for current density and the models of electron and crystal structure. And finally to understand the operation of a solar cell, we also need the wave-particle duality of electromagnetism. Thus, altogether four new cornerstones lay the foundation for the explanations of solar cell load characteristic, when one hierarchical step from the circuit model approach towards fundamental models is taken.

Now, we'll consider the cornerstones of circuit model approach in figure 3 as testable properties. In the previous chapter Kirchhoff's current law was taken as an unchallenged rule, but now the goal is to find out the sufficient conditions that validate its utilization. As already mentioned, Kirchhoff's current law arises from Maxwell's equations and the constitutive laws for current density. Hence, we end up in investigating the total electric charge. If the total charge remains constant in the region being considered, Kirchhoff's current law will be valid. But if the charge density within a region varies, the law will be violated. Consequently, Kirchhoff's current law is always valid when applied at a geometric point. But, when investigating a finite region, one has to be careful at least with the cases of high frequency. From this conclusion, we can shortly return to the title of this paper. If Kirchhoff's current law is utilized in a situation violating its prerequisites, one easily concludes: "The model works in theory, but not in practice." One of the key issues of this paper is to rethink this common statement. We try to say that incorrect results are not due to the model, and in particular, not due to mathematics. Instead, the incorrect results are due to the modeller, who was not aware of the necessary conditions validating the utilization of the model. 
Another unchallenged cornerstone in the previous section was Shockley diode equation. As the idea in this paper is not to formalize the cornerstones, we will not go into the details of deriving the equation. But still, we will take a hierarchical step towards fundamental models in order to see the cornerstones as testable properties. The Shockley diode equation describes the net motion of charges in a junction of differently doped (pn) semiconductors. In order to see underneath the Shockley diode equation, we need to go a little bit deeper into the models of solid state physics.

Generally, the operational solar cell has two necessary conditions. First, charged particles inside a solar cell absorb energy from sunlight. And second, the structure of a solar cell prevents the recombination of sunlight-induced charges.

Thus, in order to build a solar cell, we need a material, which is able to utilize the electromagnetic energy from sun. According to wave-particle duality, electromagnetic radiation can be modelled with photons, each carrying the energy inversely proportional to its wavelength. In sunlight, the wavelength of radiation is roughly between 250 and $2500 \mathrm{~nm}$, which result in photon energies approximately between 5 and $0.5 \mathrm{eV}$, respectively. Electrical insulators do not work as solar cells, since their work function, which is the minimum energy needed to release an electron in a solid material, exceeds $5 \mathrm{eV}$. In electrical conductors the value of work function is also typically close to 5 $\mathrm{eV}$, although they possess a great amount of free electrons. (Lide 2004) However, in certain semiconductors the energy of electrons increases significantly due to the sunlight exposure. Then, the absorption of photon releases an electron from the atomic bonds of crystal structure. This is why semiconductors have a significant role in the solar cell technology. Consequently, the validity of the model used in the approach of unchallenged rules requires the realization of photoelectric effect. Thus, if the cell is exposed to sunlight, then free electrons will arise.

In addition, the structure of a solar cell enables the effective utilization of sunlight-induced free charges. This is why pure semiconductors are poor solar cells. However, the junction of differently doped semiconductors is a clever solution to construct the structure preventing the recombination of sunlight-induced charges. For simplicity, let's keep the cell in the dark for now. We'll study the structure with the most common solar cell material, silicon. Pure silicon has four electrons in the outer shell, and the idea of differently doped semiconductors is to create so called n- and p-type materials. When pure silicon is doped with a material possessing more than four electrons in the outer shell, ntype is created. Phosphorus (five electrons) is typically used as a dopant. Due to doping, the work function of a semiconductor decreases significantly, since the excess electron of phosphorus atom does not contribute to bonding of atoms in crystal structure. Thus, only modest amount of external energy is required to set it free. In practice, already at room temperature the resistivity of n-type material has significantly decreased. Similarly, the idea of p-type is to dope silicon with a material possessing less than four electrons in the outer shell. Boron (three electrons) is typically used. On the contrary to n-type material, the work function of p-type semiconductor does not differ from pure silicon. However, as in n-type material, resistivity can still be decreased with a modest amount of external energy. This comes from the structure of atomic bonding in p-type material. Due to doping, some bonds are lacking an electron, and already at room temperature, this empty spot enables the net movement of electrons contributing to atomic bonds. Another important observation is that in spite of doping, the space charge density of both $\mathrm{n}$ - and p-type materials is zero, since the doping was carried out with atoms, not ions.

When these differently doped semiconductors are connected, a pn-junction is created, figure 4 . In nature, concentration differences tend to level off. At room temperature, the concentration of free electrons in n-type material is much higher than in p-type. Consequently, when a pn-junction is formed by applying a layer of n-type silicon on a p-type material, free electrons diffuse over the interface from the $\mathrm{n}$-side to the $\mathrm{p}$-side filling the empty spots of atomic bonds there. As the space 
charge densities of doped semiconductors were initially zero, the diffusion of electrons produces a net electric field over the pn-interface, which is also presented in figure 4 . This net electric field applies a drift force on electrons, the direction of which is opposite to diffusion. Thus, the rate of diffusion decreases as the electric field builds up, and finally as an equilibrium state, the depletion layer is formed as a balance between the phenomena of diffusion and drift. The electric field existing over the depletion layer, also known as built-in field, is the structure inside a silicon solar cell that prevents the recombination of light-induced free carriers by applying a net force on them. However, it is worth emphasizing that the formation of built-in field does not require the sunlight exposure. It also arises in the dark. Another important observation is that the depletion layer is empty from free charges: there are neither free electrons nor empty spots in atomic bonds.

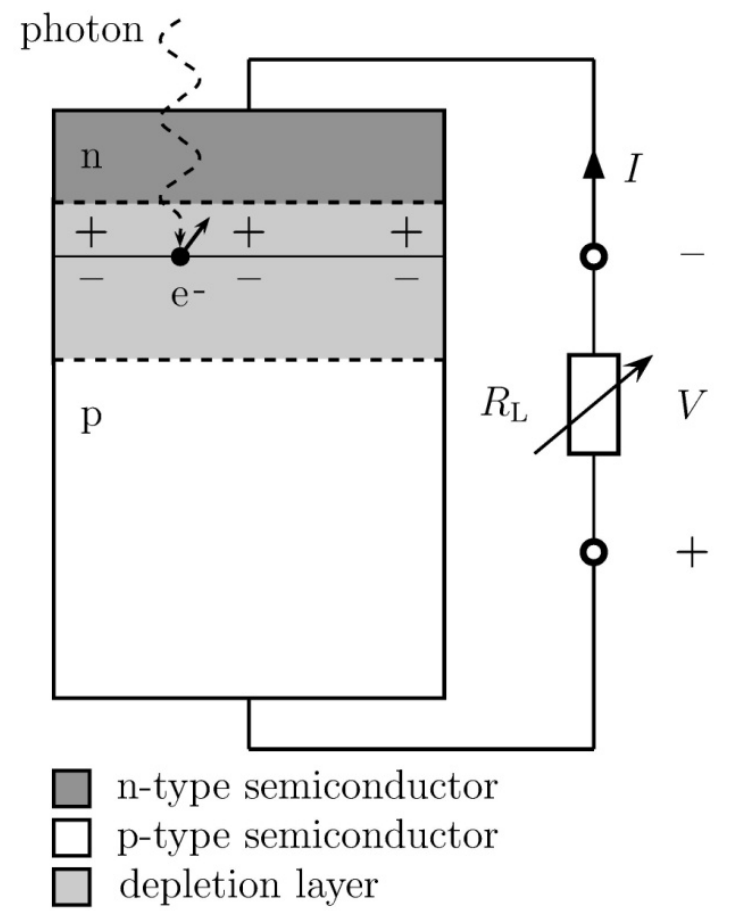

Figure 4. Photon generating a free electron e- in structural presentation of solar cell.

In order to strengthen the link between the approaches presented in figure 3, it is necessary to express the variables in equation (3) by using the terms of solid state physics. First, it has to be noticed that the short circuit current, $I_{\mathrm{sc}}$, is not included in the cornerstone of Shockley diode equation. The short circuit current, which is modelled with a current source in figure 1, represents the photon-induced current in the pn-junction. Due to the absorption of a photon in the depletion layer, free electron appears and drifts to the n-side under the influence of built-in field. Because the Shockley diode equation only models the pn-junction in the dark, the photoelectric effect is not taken into account. However, the Shockley diode equation does take into account the dark saturation current, $I_{s}$, the foundations of which are almost similar to Isc. The dark saturation current represents the drift of minority carriers in the pn-junction. Hence, if thermal energy releases an electron in the p-side of the depletion region, the built-in field drifts the free electron to the n-side. Thus, both $I_{\mathrm{sc}}$ and $I_{\mathrm{s}}$ flow in the same direction inside the pn-junction, and both the currents also represent the flow of minority carriers. The first one is modelled with the current source in the circuit of figure 1, and the latter is included in Shockley diode equation. And finally, the thermal voltage in equation (3), $V_{\mathrm{T}}$, is related to the thermal energy of electrons. As the temperature is increased, the energy of an electron increases proportional to the temperature. The thermal voltage is the voltage corresponding to this energy. 
Now, we are ready to look at the prerequisites of the Shockley diode equation. As already mentioned, it describes the net motion of charges in pn-junction. Hence, we need Maxwell's equations and the constitutive laws for current density with the following simplifying assumptions.

- The charge conservation includes the models for generation and recombination of free charges in the pn-junction.

- The constitutive laws for current density include the models of charge motion by diffusion and drift.

- There is no generation of free charges inside the pn-junction, since Shockley diode equation describes the situation in the dark. The generation of free charges due to sunlight exposure is taken into account by the means of current source in the approach of unchallenged rules.

- Inside the depletion region the space charge density is assumed to have constant positive (n) and negative (p) values. Outside the region the space charge density is zero. Furthermore, the current density in the pn-junction is assumed to consist of the diffusive flow of minority carriers only (conducting electrons in p-side and holes in n-side). Consequently, the constitutive laws for current density can be written.

These conditions will be violated for example with the high values of voltage $V$ in equation (3). As the voltage gets higher, at the same time the validity of the assumption of only minority carriers being responsible for the current density in the pn-junction, gets weaker. However, in this paper, we will not go into the detailed situations violating the Shockley equation. Instead, from the educational point of view, we want to emphasize the differences between the approaches of unchallenged rules and testable properties. In such a situation, where the measured behaviour of a solar cell differs from the modelled one, it is important that instead of judging the model as a "theoretical" one, the modeller takes responsibility of the situation by considering the cornerstones as testable properties.

\section{Discussion}

A simple circuit model approach provides a quick and cursory way to explain the solar cell load characteristic. The number of cornerstones is low and the learning time of substance for undergraduate students is few hours at most. Naturally, reliable modelling usually requires more sophisticated circuit models than the simplest one presented in figure 1. But still, in circuit analysis the cell is always considered as a black box. Consequently, this educational method certainly has its targets of application. For example, the maximum power point tracking is a topical area of solar power research, where only the solar cell terminal $I(V)$-behaviour is of interest. More generally, these kind of situations are typical for the problems related to electronics, power electronics and electric power engineering. Thus, the circuit model approach is highly valuable in multiple fields of electrical engineering.

However, if the cornerstones of modelling are taken as unchallenged rules, the connection between the model and the reality may easily remain a bit unclear. Consequently, when differences between the measured and modelled results arise, the blame is usually on "the theoretical nature of modelling". This is the main inconsistency that we want to redress with this paper. The differences between the measured and modelled results are not due to the model, and in particular, not due to mathematics. Instead, they are due to a modeller, who wasn't aware of the necessary conditions validating the utilization of a model. Thus, we want to increase the responsibility of the modeller, and as a consequence, rethink the common utterance: "In theory yes, but not in practice." Consequently, we suggest that instead of introducing modelling cornerstones to students as unchallenged rules, they should be considered as testable properties. At the same time, instead of just teaching students the 
accepted rules, they are challenged to discover things by the means of logical reasoning. Recent studies show that this kind of education may result in surprisingly good results. (Chang 2013)

This educational principle can be generalized to any topic in engineering: when students are learning the substance at a certain level of abstraction, the minimum level for a teacher is to master the conditions validating the utilization of cornerstones. Consequently, and maybe also against the general understanding, it seems that the clarity of teaching increases as the lecturer masters higher and higher levels of abstraction (Baez 2013). If the difference of profiency between the lecturer and students is low, the education will probably remain formal and unclear, at least to some extent. Thus, we suggest that from student's point of view, the unclear presentation of substance is usually related to lecturer's lack of deeper understanding. Furthermore, it has to be remembered that the individual development of profiency in natural sciences is always an endless process. So called a reductionist approach, where the pursue for deeper understanding is related to hierarchical steps towards fundamental models, offers a consistent method for a continuous development. Therefore, the educational approach that considers the cornerstones of modelling as testable properties can be considered both rewarding and demanding also for the teacher.

More generally, the hierarchical steps related to modelling in natural sciences are presented in figure 5. Common sense can be considered as the highest level of hierarchy, and when the steps of reductionism are taken downward, the level of abstraction and the delicateness of modelling increase. Each level has its own cornerstones of modelling, which lay the foundation for explanations. And when one hierarchical step towards fundamental models is taken, the cornerstones of higher level are splitted into more delicate ones. Finally, in the bottom the fundamental cornerstones of intensive research lay the foundation for modelling.

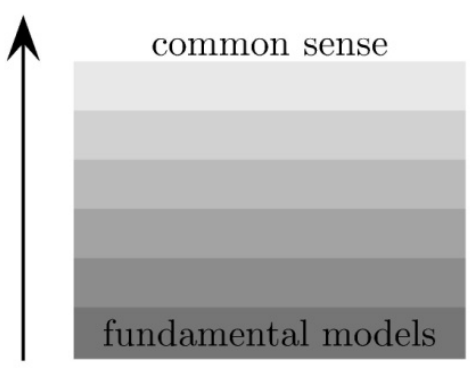

Figure 5. Hierarchical steps of modelling in natural sciences.

However, it seems that recent demands related to cost-effectiveness of universities have globally hindered a careful and time-consuming teaching of fundamentals. In addition, at least in Finland, the main focus in the development of higher education has in the current millenium been in the pedagogical skills and learning methods/environments. Certainly, these are important improvements, but they must not possess the principal trend in the development of higher education. In the end, the most efficient way to produce higher learning in higher education is to invest on the thorough learning of fundamentals. Considering the requirements on lecturers, a continuous devotion towards the deepening of substance-related profiency is called for. In the end, if graduating students "have learned to think" instead of merely accumulating information, colleges have been successful, as Derek Bok states in his famous book (Bok 2006). Furthermore, surprisingly enough, innovations and novel applications typically arise from the rock solid mastering of substance. The wise words of Sir William Henry Bragg underline the importance of deeper comprehension in the emerge of innovations: "The important thing in science is not so much to obtain new facts as to discover new ways of thinking about them." (Mishra 2006) 


\section{Conclusions}

This paper originated from the honest concern related to higher education in engineering. Mainly due to present economic pressures that universities live in, a global and increasing problem has arisen: there is not enough higher learning in higher education. In engineering higher learning is essentially related to thorough understanding of natural phenomena which, in turn, requires a careful study of fundamentals. Thus, in order to achieve deeper understanding, learning of the fundamentals in any branch of science deserves a special attention.

As a result of this paper, we suggest that despite the level of abstraction, special caution should be paid on the conditions validating the modelling. To clarify the idea, an educational case study related to solar cell load characteristic was carried out in two different ways. First, the explanations for the solar cell behaviour were based on the unchallenged cornerstones of modelling. Then, a hierarchical step towards fundamental models was taken in order to look underneath the cornerstones. Consequently, instead of unchallenged rules, the cornerstones were seen as testable properties. An important difference between these educational methods is the increased responsibility of the modeller. If the cornerstones are taken as unchallenged rules, the differences between measured and modelled results will be easily judged as a consequence of "theoretical" models, which are not "practical". But when the cornerstones are seen as testable properties, a modeller realizes that the practicality of modelling is essentially related to the validity of necessary conditions of cornerstones. Thus, a modeller takes more responsibility on modelling. And consequently, the common utterance "in theory yes, but not in practice" becomes mostly irrelevant.

In engineering, despite the deepness of comprehension, the personal substance-related progress is always an endless process. For a student, the utilization of cornerstones offers a consistent tool to deepen ones understanding. But furthermore, also lecturers gain from a reductionist approach, since the demandingness of education rises with the increasing level of abstraction. Lecturer's ability to clearly express the necessary conditions related to the validity of cornerstones can be considered as a proof of proficiency. On the other hand, also the lecturer's shortages of substance become transparent. Therefore, the utilization of cornerstones in engineering education is both profitable and demanding for the lecturer.

The development of technology is fast. Technical devices and their designing principles easily expire in a decade. Therefore, in addition to topical short-term engineering skills, higher education should pay special attention on the permanent engineering skills, which are essentially related to a deeper comprehension of natural phenomena and fundamental models. Regardless of technical development, the usefulness related to the deeper understanding of natural phenomena remains. Furthermore, in engineering education, instead of teaching the formal accepted rules, students should be given a position enabling them to add new and more accurate knowledge directly above the previously adopted view. In the end, we are dealing with a serious matter that should be extensively understood in university administrations, since its consequences are far-reaching.

\section{References}

Anderson, P. W. (1972). More Is Different. Science, vol. 177, pp. 393-396.

Baez, J. C. (2013). Levels of excellence. The blog of Azimuth Project, September 29, johncarlosbaez.wordpress.com.

Benezet, L. P. (1935). The Teaching of Arithmetic I, The Story of an Experiment. Journal of the National Education Association, vol. 24, pp. 241-244.

Benezet, L. P. (1935). The Teaching of Arithmetic II, The Story of an Experiment. Journal of the National Education Association, vol. 24, pp. 301-303.

Benezet, L. P. (1936). The Teaching of Arithmetic III, The Story of an Experiment. Journal of the National Education Association, vol. 25, pp. 7-8.

Bok, D. (2003). Universities in the Marketplace. Princeton University Press.

Bok, D. (2006). Our Underachieving Colleges. Princeton University Press. 
Bloom, B. S., Engelhart, M. D., Furst, E. J., Hill, W. H. \& Krathwohl, D. R. (1956). Taxonomy of educational objectives: Handbook I: Cognitive domain!. New York: David McKay Company.

Chang, K. (2013). Expecting the Best Yields Results in Massachusetts. The New York Times, September 2.

Feynman, R. P., Leighton, R. B., Sands, M. \& Treiman, S. B. (1963). The Feynman Lectures on Physics. Addison-Wesley.

Keeling, R. P. \& Hersh, R. H. (2011). We Are Losing Our Minds, Rethinking American Higher Education. Keeling \& Associates, LLC.

Lide, D. R. (2004). CRC Handbook of Chemistry and Physics. CRC press.

Mishra, P. \& Koehler, M. J. (2006). Technological Pedagogical Content Knowledge: A Framework for Teacher Knowledge. Teachers College Record, vol. 108, pp. 1017-1054.

Polkinghorne, J. (2002). Reductionism. Interdiciplinary Encyclopedia of Religion and Science. www.inters.org.

Rosovsky, H. (1990). The University, an Owner's Manual. W. W. Norton \& Company.

Sheppard, S. D., Macatangay, K., Colby, A. \& Sullivan W. M. (2008). Educating Engineers, Designing for the Future of the Field. Carnegie/Jossey-Bass.

Shulman, L. S. (1972). Those Who Understand: Knowledge Growth in Teaching. Educational Researcher, vol. 12, pp. 4-14.

Sullivan, W. M. \& Rosin, M. S. (2008). A New Agenda for Higher Education, Shaping a Life of the Mind for Practise. Carnegie/JosseyBass.

The Economist. (2012). Not what it used to be. Dec 1st, Chicago.

The New York Times. (1986). About Education: Learning Math by Thinking, June 10.

Tinkham, M. (1975). Introduction to superconductivity. McGraw-Hill.

Whitney, H. (1985). Taking Responsibility in School Mathematics Education. The Journal of Mathematical Behavior, vol. 4, pp. 219-235.

Whitney, H. (1987). Coming Alive in School Math and Beyond. Educational Studies in Mathematics, vol. 18, pp. 229-242.

Wieman, C. E. (2013). Ideas for Improving Science Education. The New York Times, September 2. 